\title{
Cryo-EM structure of an amyloid fibril formed by full-length human SOD1 reveals its pathological conformational conversion
}

\author{
Li-Qiang Wang ${ }^{1,2,9}$, Yeyang $\mathrm{Ma}^{3,4,9}$, Han-Ye Yuan ${ }^{1,2,9}$, Kun Zhao ${ }^{3,4}$, Mu-Ya
}

Zhang $^{1,2}$, Qiang Wang ${ }^{5}$, Xi Huang ${ }^{6}$, Wen-Chang $\mathrm{Xu}^{1}$, Jie Chen ${ }^{1,2}$, Dan $\mathrm{Li}^{7,8}$, Delin

Zhang $^{5}$, Liangyu Zou ${ }^{6}$, Ping Yin ${ }^{5}$, Cong Liu ${ }^{3,10 \bowtie} \&$ Yi Liang ${ }^{1,2,10 \bowtie ~}$

${ }^{1}$ Hubei Key Laboratory of Cell Homeostasis, College of Life Sciences, Wuhan University, Wuhan 430072, China

${ }^{2}$ Wuhan University Shenzhen Research Institute, Shenzhen 518057, China

${ }^{3}$ Interdisciplinary Research Center on Biology and Chemistry, Shanghai Institute of Organic Chemistry, Chinese Academy of Sciences, Shanghai 201210, China

${ }^{4}$ University of Chinese Academy of Sciences, Beijing 100049, China

${ }^{5}$ National Key Laboratory of Crop Genetic Improvement and National Centre of Plant Gene Research, Huazhong Agricultural University, Wuhan 430070, China

${ }^{6}$ Department of Neurology, the Second Clinical Medical College, Jinan University (Shenzhen People's Hospital), Shenzhen 518020, China

${ }^{7}$ Key Laboratory for the Genetics of Developmental and Neuropsychiatric Disorders, Ministry of Education, Bio-X Institutes, Shanghai Jiao Tong University, Shanghai 200030, China

${ }^{8}$ Zhangjiang Institute for Advanced Study, Shanghai Jiao Tong University, Shanghai 200240, China

${ }^{9}$ These authors contributed equally: Li-Qiang Wang, Yeyang Ma, Han-Ye Yuan.

${ }^{10}$ These authors jointly supervised this work: Cong Liu, Yi Liang. ${ }^{\mathrm{e}-m a i l}$ : 
bioRxiv preprint doi: https://doi.org/10.1101/2021.12.07.471532; this version posted December 9, 2021. The copyright holder for this preprint

(which was not certified by peer review) is the author/funder, who has granted bioRxiv a license to display the preprint in perpetuity. It is made available under aCC-BY-NC-ND 4.0 International license.

liulab@sioc.ac.cn; liangyi@whu.edu.cn

Correspondence and requests for materials should be addressed to C.L. or Y.L. 
Amyotrophic lateral sclerosis (ALS) is a progressive, fatal neurodegenerative disease characterized by the selective death of motor neurons. Misfolded $\mathrm{Cu}, \mathrm{Zn}$-superoxide dismutase (SOD1) has been linked to both familial ALS and sporadic ALS. SOD1 fibrils formed in vitro are able to incorporate into cells, transmit intercellularly, and share toxic properties with ALS inclusions. Here we produced amyloid fibrils in vitro from recombinant, full-length apo human SOD1 under semi-reducing conditions and determined the atomic structure using cryo-EM. The SOD1 fibril consists of a single protofibril with a left-handed helix. The fibril core exhibits a serpentine fold comprising $\mathrm{N}$-terminal segment (residues 3 to 55) and C-terminal segment (residues 86 to 153 ) with a structural break. The two segments are zipped up by three salt bridge pairs. By comparison with the structure of apo SOD1 dimer, we propose that eight $\beta$-strands (to form a $\beta$-barrel) and one $\alpha$-helix in the subunit of apo SOD1 convert into thirteen $\beta$-strands stabilized by five hydrophobic cavities in the SOD1 fibril. Our data provide insights into how SOD1 converts between structurally and functionally distinct states. 
Amyotrophic lateral sclerosis (ALS), also called Lou Gehrig's disease, is a neurodegenerative disease that involves the loss of upper and lower motor neurons ${ }^{1-8}$. Ninety percent of ALS cases are sporadic and little is known about the origin, while ten percent of ALS cases are inherited familial $\mathrm{ALS}^{2-9}$. The sodl gene, serving as a major antioxidant gene, was the first to be linked to the familial form of $\mathrm{ALS}^{1}$ and other genes associated with genetic ALS include those encoding TDP-43 and FUS ${ }^{2,7}$. The misfolding of human $\mathrm{Cu}, \mathrm{Zn}$-superoxide dismutase (SOD1) in motor neuron cells play a crucial role in etiology of the disease ${ }^{1-9}$. Misfolded SOD1 aggregates were widely observed in the spinal cords of both genetic ALS and sporadic ALS cases ${ }^{5-9}$. The functional human SOD1 is a 32-kDa homo-dimeric metalloenzyme; each subunit consists of 153 amino acid residues and contains one copper ion and one zinc ion ${ }^{10-13}$. The SOD1 structure in each subunit features an antiparallel $\beta$-barrel composed of eight $\beta$-strands and two $\alpha$-helices, which is stabilized by a disulfide bond between $\mathrm{Cys}^{57}$ and $\mathrm{Cys}^{146}$ (refs. ${ }^{12,13}$ ). In sharp contrast, the high-resolution structures of amyloid fibrils formed by full-length ALS-associated proteins including SOD1, TDP-43, and FUS are not available so far $^{2,14-19}$. Therefore, it is unclear for the conformational conversion of SOD1 from its mature form into an aggregated form during pathogenesis of ALS.

The mature form of SOD1 is exceptionally stable and unlikely to be a candidate for protein aggregation ${ }^{10,11,20}$. Instead, it has been proposed that immature forms of SOD1, which lack copper and zinc ions and the disulfide bond in the structure, are the origin for cytotoxic misfolded conformations ${ }^{10,11,20-22}$. Previous studies have shown that the 
amyloid-like aggregates isolated from ALS transgenic mice or cells expressing SOD1 mutations causing ALS contain metal-deficient and disulfide-reduced SOD1, suggesting their pathogenic potential ${ }^{23-25}$. Substantial experiments have demonstrated that the full-length apo SOD1 can convert into amyloid fibrils by incubation with reducing agents in vitro ${ }^{26-33}$. Importantly, the SOD1 fibrils formed in vitro are able to incorporate into cells and transmit intercellularly ${ }^{9}$. Moreover, SOD1 fibrils produced under reducing conditions also share pathological properties with ALS inclusions, such as the abilities to induce mitochondria damage, cause neuroinflammation and activate microglial cells triggering neurodegeneration in $\operatorname{ALS}^{34-36}$. Thus, structural determination of the SOD1 fibrils is of importance for understanding the pathogenic mechanism of SOD1 in both familial ALS and sporadic ALS.

Here we prepared homogeneous amyloid fibrils in vitro from recombinant, full-length apo human SOD1 under reducing conditions and determine the atomic structure by using cryo-EM. Our findings provide structural insights into the conversion of SOD1 between physiological and pathological states.

\section{Results}

SOD1 forms amyloid fibrils under semi-reducing conditions. Treatment of the apo form of SOD1 with 5-10 mM tris (2-carboxyethyl) phosphine (TCEP), a highly stable disulfide-reducing agent, generates a semi-reduced state that is able to mimic the gradual reduction of apo SOD1 occurring intracellularly upon loss of copper and zinc ions $^{20,21,26,30,32,33}$. We produced amyloid fibrils from recombinant, full-length apo 
human SOD1 (residues 1 to 153) overexpressed in Escherichia coli, by incubating the purified apo protein in $20 \mathrm{mM}$ tris- $\mathrm{HCl}$ buffer (pH 7.4) containing $5 \mathrm{mM}$ TCEP and shaking at $37{ }^{\circ} \mathrm{C}$ for $40-48 \mathrm{~h}$ (see methods). SOD1 fibrils formed under such semi-reducing conditions were concentrated to $\sim 30 \mu \mathrm{M}$ in a centrifugal filter (Millipore) and examined by electron microscopy without further treatment.

Negative-staining transmission electron microscopy (TEM) imaging showed that recombinant, full-length apo SOD1 formed homogeneous and unbranched fibrils under semi-reducing conditions (Extended Data Fig. 1a). The SOD1 fibril is composed of a single protofibril with a fibril full width of $11.3 \pm 1.0 \mathrm{~nm}(n=8)$ (Extended Data Fig. 1a), which is consistent with previously described in vitro amyloid fibrils produced from full-length apo SOD1 under the same semi-reducing conditions, which showed a width of $\sim 11 \mathrm{~nm}$ based on negative staining on $\mathrm{TEM}^{32}$. Congo red binding assays showed a red shift of the maximum absorbance, from 490 to $550 \mathrm{~nm}$, in the presence of SOD1 fibrils (Extended Data Fig. 1b), which is the characteristics of amyloid fibrils ${ }^{37,38}$. Together, the data showed that full-length apo SOD1 forms typical amyloid fibrils under semi-reducing conditions.

Cryo-EM structure of SOD1 fibrils. We next determined the atomic structure of the SOD1 amyloid fibrils by cryo-EM (Table 1 and Figs. 1 and 2). The cryo-EM micrographs and two-dimensional (2D) class average images show that the SOD1 fibril is composed of a single protofibril with a left-handed helical twist (Fig. 1a and Extended Data Fig. 2a). The helical pitch is $144 \pm 5 \mathrm{~nm}$ (Fig. 1a). The SOD1 subunit 
within the protofibril is arranged in a staggered manner (Extended Data Fig. 2b). The fibrils are morphologically homogeneous, showing a fibril full width of $12.3 \pm 0.7 \mathrm{~nm}$ (Fig. 1a and Extended Data Fig. 2a).

Using helical reconstruction in RELION3.1 (ref. ${ }^{39}$ ), we determined a density map of the ordered core of SOD1 fibrils, with an overall resolution of $3.08 \AA$, which features well-resolved side-chain densities and clearly separated $\beta$ strands along the fibril axis (Fig. 1b and Extended Data Fig. 3). Cross-sectional view of the 3D map of the SOD1 fibril and top view of the density map show a protofibril comprising the $\mathrm{N}$ and the C-terminal segments, with an unstructured flexible region in between (Fig. 1b,e). The 3D map showed a single protofibril in the SOD1 fibril with a left-handed helix. The half-helical pitch is $73.1 \mathrm{~nm}$ (Fig. 1c). The SOD1 subunit within the protofibril stacks along the fibril axis with a helical rise of $4.82 \AA$ and twist of $-1.187^{\circ}$ (Fig. 1d).

We unambiguously built a structure model of SOD1 fibril comprising the N-terminal segment (residues 3 to 55) and the C-terminal segment (residues 86 to 153) at $3.08 \AA$ (Fig. 2). The electron density of the structural break comprising residues 56 to 85 is invisible due to high flexibility (Fig. 2a-c), which is reminiscent of the breaks observed in the structures of patient-derived amyloid fibrils from systemic AL amyloidosis $^{40,41}$. The presence of a structural break represents an interesting structural feature of SOD1 fibrils formed under semi-reducing conditions.

Side chains for the residues in the SOD1 fibril core can be well accommodated into the density map (Fig. 2a). The exterior of the SOD1 fibril core is mostly hydrophilic, 
whereas side chains of most hydrophobic residues are located in the interior of the SOD1 fibril fold (Fig. 2b-g and Extended Data Fig. 4). Five hydrophobic cavities (Extended Data Fig. 4a and Fig. 2g), four hydrogen bonds (Extended Data Fig. 5a), and a very compact fold (Fig. 2b,d) help stabilize the fibril core, as described in detail below.

Hydrophobic side chains of Val5, Leu8, Val14, and $\mathrm{Ile}^{17}$, hydrophobic side chains of Ile18, Phe20, Leu42, Phe45, Val47, and Phe50, and hydrophobic side chains of Va129, Leu31, Ile35, and Leu38 are located in the interior of the N-terminal part of SOD1 fibril to form three hydrophobic cavities (Extended Data Fig. 4b-d), and hydrophobic side chains of Val87, Ala89, and Ala95 and hydrophobic side chains of Va194, Va197, Ile99, Ile104, Leu106, Ile113, Leu144, Ala145, Val148, Ile149, Ile151, and Ala152 are located in the interior of the C-terminal part to form two hydrophobic cavities (Extended Data Fig. 4e-f), thereby stabilizing the SOD1 fibrils. Two pairs of amino acids (Val14 and Asn53; and Gln15 and Phe50) from the N-terminal segment (Extended Data Fig. 5b) and two pairs of amino acids (Gly130 and Asp125; and Arg143 and Gln153) from the C-terminal segment (Extended Data Fig. 5c,d) form four hydrogen bonds to stabilize the fibril core.

The SOD1 fibril core features a very compact fold containing thirteen $\beta$-strands ( $\beta 1$ to $\beta 13$ ) and an in-register intramolecular $\beta$-strand architecture (Fig. 2b,d). Six $\beta$-strands ( $\beta 1$ to $\beta 6)$ and seven $\beta$-strands $(\beta 7$ to $\beta 13)$ are present in the $N$ - and C-terminal segments of the SOD1 fibril core structure, respectively (Fig. 2b-d). The height of one layer along the helical axis is $15.82 \AA$, which is the distance between the 
highest point in the loop between $\beta 4$ and $\beta 5$ and the lowest point in the loop between $\beta 8$ and $\beta 9$ (Fig. 2e).

The SOD1 fibril contains a long intramolecular interface comprising residues 36 to 48 in the N-terminal half and residues 98 to 109 in the C-terminal half (Fig. 3a). Three pairs of intramolecular salt bridges formed by Lys36 and Asp109, His43 and Asp101, and His46 and Glu100 (with distances less than $4 \AA$ A; Fig. 3b-e) are identified to stabilize the intramolecular L-shaped interface between the $\mathrm{N}$ - and C-terminal parts of SOD1 fibril (Figs. 2a,b,f,g and 3b-e). The intramolecular L-shaped interface in the SOD1 fibril features mixed compositions of hydrophilic and hydrophobic side chains, whereas side chains of most residues in the interior of such an interface are hydrophilic (Fig. 2b-g). The presence of a mostly hydrophilic intramolecular interface represents another interesting structural feature of SOD1 fibrils formed under semi-reducing conditions.

\section{Discussion}

We compared the structures of apo SOD1 dimer and SOD1 fibrils produced under semi-reducing conditions (Fig. 4). Notably, the SOD1 molecule adopts largely distinctive secondary, tertiary, and quaternary structures in two different states of SOD1, highlighting the high structural polymorphs and phenotypic diversity of SOD1 in physiological and pathological states. The apo human SOD1 dimer contains eight $\beta$-strands (to form an antiparallel $\beta$-barrel), two $\alpha$-helices, and a single disulfide bond between Cys57 in $\alpha 1$ and Cys146 in $\beta 8^{\prime}$ in each subunit as well as an intermolecular 
interface involving strong hydrophobic interactions and hydrogen bonding from Gly51 and Gly114 of one molecule to Ile151 of the other ${ }^{12}$ (Fig. 4a,b). In contrast, once folding into fibril structure, SOD1 molecules forms six $\beta$-strands $(\beta 1$ to $\beta 6)$ by its $\mathrm{N}$-terminal segment (residues 3 to 55 ) and seven $\beta$-strands ( $\beta 7$ to $\beta 13$ ) by its C-terminal segment (residues 86 to 153 ), exhibiting an in-register intramolecular $\beta$ strand architecture (Fig. 4a,c). Moreover, the SOD1 fibril structure features a long, mostly hydrophilic intramolecular L-shaped interface and a structural break comprising residues 56 to 85 (Fig. 4a,c). Once apo SOD1 dimer converts into its fibrillar form, the SOD1 molecule undergoes a completely conformational rearrangement, with the antiparallel $\beta$-barrel of apo SOD1 converted to $\beta 1-\beta 5, \beta 7, \beta 8$, $\beta 11$, and $\beta 13$, the loop between $\beta 4^{\prime}$ and $\alpha 1$ converted to $\beta 6, \alpha 1$ and the loop between $\alpha 1$ and $\beta 5^{\prime}$ converted to the structural break, the loop between $\beta 6^{\prime}$ and $\beta 7^{\prime}$ 'converted to $\beta 9$ and $\beta 10$, and $\alpha 2$ of apo SOD1 converted to $\beta 12$ in the SOD1 fibril (Fig. $4 b, c)$.

Previous studies proposed two alternative models of SOD1 fibrils based on protease digestion experiments and mass spectrometric analyses ${ }^{28,29}$. The so-called "three key region model" predicts that the SOD1 fibril core contains one N-terminal segment comprising residues 1 to 30 and two $\mathrm{C}$-terminal segments comprising residues $90-120$ and $135-153$ (ref. ${ }^{28}$ ). This is in good agreement with our model, wherein $\beta 1$ and $\beta 2, \beta 8$ to $\beta 11$, and $\beta 13$ would correspond to the first, second, and third segments in the three key region $\operatorname{model}^{28}$. The other $\mathrm{N}$-terminal core model predicts that the SOD1 fibril core contains the first 63 residues of the $\mathrm{N}$ terminus of the protein ${ }^{29}$. This is partly compatible with our model, wherein the six $\beta$ strands ( $\beta 1$ 
to $\beta 6$ ) present in the $\mathrm{N}$-terminal segment would correspond to the minimal protease-resistant core region comprising residues 1 to 63 in the $\mathrm{N}$-terminal core model $^{29}$. In all three models, SOD1 fibrils are produced from the immature form of the protein under semi-reducing conditions, in order to mimic the conditions of the gradual reduction of apo SOD1 occurring intracellularly upon loss of metal ions $^{20,21,26,28-33}$. Previous work had shown that amyloid fibril formation is initiated by the immature, disulfide-reduced, apo form of SOD1 (ref. ${ }^{26}$ ). In our SOD1 fibril model, Cys57, Cys111, and Cys146 are all in disulfide-reduced conformations with free thiol groups. Intriguingly, the side chains of those Cys residues appears exposed to quite crowded areas of the first and fifth hydrophobic cavities (Fig. 2a,b and Extended Data Fig. 4b,f).

Strikingly, among two hundred and seventeen genetic mutations of SOD1 identified from different familial $\mathrm{ALS}^{1-5,8,20-25,28,29,32,42-47}$ (https://alsod.iop.kcl.ac.uk/), one hundred and eighty-two clinically identified mutations are located within the SOD1 fibril core structure determined in this study, in which one hundred and five representative genetic ALS-related mutations are listed in Fig. 4a. Notably, residues forming strong salt bridges (His43, His46, Glu100, Asp101, and Asp109) that contribute to stabilization of the intramolecular L-shaped interface between the $\mathrm{N}$ and C-terminal parts of SOD1 fibril (Figs. 2a,b,f,g and 3b-e) or hydrogen bonds (Val14, Asp125, and Gln153) that contribute to maintenance of the SOD1 fibril structure (Extended Data Fig. 5b-d) are also ALS-associated mutation sites $^{1-5,8,20-25,28,29,32,42-47}$. Based on the cryo-EM fibril structure, the disease mutations, 
such as H43R, H46R, H46D, E100G, E100K, D101G, D101H, D101N, D101Y, D109Y, D109N, and D125H (Fig. 4a, salt bridge mutations and hydrogen bond mutations), may disrupt important interactions in the SOD1 fibril structure. This suggests that the different mutations may induce SOD1 to form fibrils with structures distinct from the one presented here, which might be related to structural diversity of SOD1 fibrils, strains, and phenotypic diversity of SOD1 in pathological state ${ }^{2,26,28}$. Interestingly, as for the ALS-associated residues including His43, His46, Glu100, Asp101, and Asp109, His43 forms a hydrogen bond with Thr39, His46 forms a strong salt bridge with Asp124, and Asp101 forms a strong salt bridge with Arg79 in the subunit to stabilize the structure of apo SOD1 dimer (Extended Data Fig. 6a-d), whereas His43 and Asp101 form a strong salt bridge and His46 forms a strong salt bridge with Glu100 in the SOD1 fibril to stabilize the intramolecular L-shaped interface (Fig. 3b-d). This indicates that reorganization of salt bridges may occur for these ALS-associated mutated SOD1 during their conformational conversion from apo into fibrillar form.

In summary, we revealed by cryo-EM that the full-length human SOD1 displays a novel amyloid fibril structure. The SOD1 fibril displays a very compact fold with a structural break, which contains thirteen $\beta$-strands stabilized by five hydrophobic cavities and four hydrogen bonds, and a long, mostly hydrophilic intramolecular L-shaped interface stabilized by three strong salt bridges. The comparison of the structures of apo SOD1 dimer and SOD1 fibril reveals the substantial conformational conversion from a $\beta$-sheet-rich (correspond to the antiparallel $\beta$-barrel structure), 
immature form of SOD1 to a totally distinct $\beta$-sheet-rich (correspond to an in-register intramolecular $\beta$ strand architecture), fibrillar form of SOD1 during pathogenesis of ALS. The fibril structure will be valuable in regard to understanding the structural basis underlying SOD1 misfolding and inspiring future research on the structural polymorphism of SOD1 strains and their relationship to ALS.

1. Rosen, D. R. et al. Mutations in $\mathrm{Cu} / \mathrm{Zn}$ superoxide dismutase gene are associated with familial amyotrophic lateral sclerosis. Nature 362, 59-62 (1993).

2. Ayers, J. I. \& Borchelt, D. R. Phenotypic diversity in ALS and the role of poly-conformational protein misfolding. Acta Neuropathol. 142, 41-55 (2021).

3. Shaw, B. F. \& Valentine, J. S. How do ALS-associated mutations in superoxide dismutase 1 promote aggregation of the protein? Trends. Biochem. Sci. 32, 78-85 (2007).

4. Valentine, J. S., Doucette, P. A. \& Potter, S. Z. Copper-zinc superoxide dismutase and amyotrophic lateral sclerosis. Annu. Rev. Biochem. 74, 563-593 (2005).

5. Chattopadhyay, M. \& Valentine, J. S. Aggregation of copper-zinc superoxide dismutase in familial and sporadic ALS. Antioxid. Redox Signal. 11, 1603-1614 (2009).

6. Xu, W. C. et al. Pathological hydrogen peroxide triggers the fibrillization of wild-type SOD1 via sulfenic acid modification of Cys-111. Cell Death Dis. 9, 67 (2018).

7. Polymenidou, M. \& Cleveland, D. W. The seeds of neurodegeneration: prion-like 
spreading in ALS. Cell 147, 498-508 (2011).

8. Cleveland, D. W. \& Rothstein J. D. From Charcot to Lou Gehrig: deciphering selective motor neuron death in ALS. Nat. Rev. Neurosci. 2, 806-819 (2001).

9. GradL. I. et al. Intercellular propagated misfolding of wild-type $\mathrm{Cu} / \mathrm{Zn}$ superoxide dismutase occurs via exosome-dependent and -independent mechanisms. Proc. Natl. Acad. Sci. U.S.A. 111, 3620-3625 (2014).

10. Sala F, A., Wright, G. S. A., Antonyuk, S. V., Garratt, R. C. \& Hasnain, S. S. Molecular recognition and maturation of SOD1 by its evolutionarily destabilised cognate chaperone hCCS. PLoS Biol. 17, e3000141(2019).

11. Luchinat, E. \& Banci, L. In-cell NMR in human cells: direct protein expression allows structural studies of protein folding and maturation. Acc. Chem. Res. 51, 1550-1557 (2018).

12. Strange, R. W. et al. The structure of holo and metal-deficient wild-type human $\mathrm{Cu}, \mathrm{Zn}$ superoxide dismutase and its relevance to familial amyotrophic lateral sclerosis. J. Mol. Biol. 328, 877-891 (2003).

13. Banci, L. et al. Structural and dynamic aspects related to oligomerization of apo SOD1 and its mutants. Proc. Natl. Acad. Sci. U.S.A. 106, 6980-6985 (2009).

14. Iwakawa, N., Morimoto, D., Walinda, E., Shirakawa, M. \& Sugase, K. Multiple-state monitoring of SOD1 amyloid formation at single-residue resolution by Rheo-NMR spectroscopy. J. Am. Chem. Soc. 143, 10604-10613 (2021).

15. Sangwan, S. et al. Atomic structure of a toxic, oligomeric segment of SOD1 linked to amyotrophic lateral sclerosis (ALS). Proc. Natl. Acad. Sci. U.S.A. 114, 
8770-8775 (2017).

16. Ivanova, M. I. et al. Eisenberg, Aggregation-triggering segments of SOD1 fibril formation support a common pathway for familial and sporadic ALS. Proc. Natl. Acad. Sci. U.S.A. 111, 197-201 (2014).

17. Murray, D. T. et al. Structure of FUS protein fibrils and its relevance to self-assembly and phase separation of low-complexity domains. Cell 171, 615-627 (2017).

18. Lee, M., Ghosh, U., Thurber, K. R., Kato, M. \& Tycko, R. Molecular structure and interactions within amyloid-like fibrils formed by a low-complexity protein sequence from FUS. Nat. Commun. 11, 5735 (2020).

19. Li, Q., Babinchak, W. M. \& Surewicz, W. K. Cryo-EM structure of amyloid fibrils formed by the entire low complexity domain of TDP-43. Nat. Commun. 12, 1620 (2021).

20. Tiwari, A., Xu, Z. \& Hayward, L. J. Aberrantly increased hydrophobicity shared by mutants of $\mathrm{Cu}, \mathrm{Zn}$-superoxide dismutase in familial amyotrophic lateral sclerosis. J. Biol. Chem. 280, 29771-29779 (2005).

21. Tiwari, A. \& Hayward, L. J. Familial amyotrophic lateral sclerosis mutants of copper/zinc superoxide dismutase are susceptible to disulfide reduction. J. Biol. Chem. 278, 5984-5992 (2003).

22. Furukawa, Y., Kaneko, K., Yamanaka, K., O’Halloran, T. V. \& Nukina, N. Complete loss of post-translational modifications triggers fibrillar aggregation of SOD1 in the familial form of amyotrophic lateral sclerosis. J. Biol. Chem. 283, 
24167-24176 (2008).

23. Jonsson, P. A. et al. Disulphide-reduced superoxide dismutase-1 in CNS of transgenic amyotrophic lateral sclerosis models. Brain 129, 451-464 (2006).

24. Shaw, B. F. et al. Detergent-insoluble aggregates associated with amyotrophic lateral sclerosis in transgenic mice contain primarily full-length, unmodified superoxide dismutase-1. J. Biol. Chem. 283, 8340-8350 (2008).

25. Bourassa, M. W., Brown, H. H., Borchelt, D. R., Vogt, S. \& Miller, L. M. Metal-deficient aggregates and diminished copper found in cells expressing SOD1 mutations that cause ALS. Front. Aging. Neurosci. 6, 110 (2014).

26. Chattopadhyay, M. et al. Initiation and elongation in fibrillation of ALS-linked superoxide dismutase. Proc. Natl. Acad. Sci. U.S.A. 105, 18663-18668 (2008).

27. Rousseau, F., Schymkowitz, J. \& Oliveberg, M. ALS precursor finally shaken into fibrils. Proc. Natl. Acad. Sci. U.S.A. 105, 18649-18650 (2008).

28. Furukawa, Y., Kaneko, K., Yamanaka, K. \& Nukina, N. Mutation-dependent polymorphism of $\mathrm{Cu}, \mathrm{Zn}$-superoxide dismutase aggregates in the familial form of amyotrophic lateral sclerosis. J. Biol. Chem. 285, 22221-22231 (2010).

29. Chan, P. K. et al. Structural similarity of wild-type and ALS-mutant superoxide dismutase-1 fibrils using limited proteolysis and atomic force microscopy. Proc. Natl. Acad. Sci. U.S.A. 110, 10934-10939 (2013).

30. Chattopadhyay, M. et al. The disulfide bond, but not zinc or dimerization, controls initiation and seeded growth in amyotrophic lateral sclerosis-linked $\mathrm{Cu}, \mathrm{Zn}$ superoxide dismutase (SOD1) fibrillation. J. Biol. Chem. 290, 30624-30636 
(2015).

31. Khan, M. A. I. et al. $\mathrm{Cu} / \mathrm{Zn}$ superoxide dismutase forms amyloid fibrils under near-physiological quiescent conditions: the roles of disulfide bonds and effects of denaturant. ACS Chem. Neurosci. 8, 2019-2026 (2017).

32. Koo, B. K., Munroe W., Gralla E. B., Valentine J. S. \& Whitelegge J. P. A novel SOD1 intermediate oligomer, role of free thiols and disulfide exchange. Front. Neurosci. 14, 619279 (2021).

33. Abdolvahabi, A., Shi, Y., Chuprin, A., Rasouli, S. \& Shaw, B. F. Stochastic formation of fibrillar and amorphous superoxide dismutase oligomers linked to amyotrophic lateral sclerosis. ACS Chem. Neurosci. 7, 799-810 (2016).

34. Salehi, M., Nikkhah, M., Ghasemi, A. \& Arab, S. S. Mitochondrial membrane disruption by aggregation products of ALS-causing superoxide dismutase-1 mutants. Int. J. Biol. Macromol. 75, 290-297 (2015).

35. Fiala, M. et al. IL-17A is increased in the serum and in spinal cord CD8 and mast cells of ALS patients. J. Neuroinflammation 7, 76 (2010).

36. Roberts, K. et al. Extracellular aggregated $\mathrm{Cu} / \mathrm{Zn}$ superoxide dismutase activates microglia to give a cytotoxic phenotype. Glia 61, 409-419 (2013).

37. Wang, L. Q. et al. Cryo-EM structure of an amyloid fibril formed by full-length human prion protein. Nat. Struct. Mol. Biol. 27, 598-602 (2020).

38. Wang, L. Q. et al. Genetic prion disease-related mutation E196K displays a novel amyloid fibril structure revealed by cryo-EM. Sci. Adv. 7, eabg9676 (2021). 
39. Scheres, S. H. W. Amyloid structure determination in RELION-3.1. Acta Crystallogr. D Struct. Biol. 76, 94-101 (2020).

40. Radamaker, L. et al. Cryo-EM reveals structural breaks in a patient-derived amyloid fibril from systemic AL amyloidosis. Nat. Commun. 12, 875 (2021).

41. Radamaker, L. et al. Role of mutations and post-translational modifications in systemic AL amyloidosis studied by cryo-EM. Nat. Commun. 12, 6434 (2021).

42. Arisato, T. et al. Clinical and pathological studies of familial amyotrophic lateral sclerosis (FALS) with SOD1 H46R mutation in large Japanese families. Acta Neuropathol. 106, 561-568 (2003).

43. Cudkowicz, M. E. et al. Epidemiology of mutations in superoxide dismutase in amyotrophic lateral sclerosis. Ann. Neurol. 41, 210-221 (1997).

44. Prudencio, M., Hart P. J., Borchelt D. R. \& Andersen P. M. Variation in aggregation propensities among ALS-associated variants of SOD1: correlation to human disease, Hum. Mol. Genet. 18, 3217-3226 (2009).

45. Sandelin, E., Nordlund, A., Andersen, P. M., Marklund, S. S. L. \& Oliveberg, M. Amyotrophic lateral sclerosis-associated copper/zinc superoxide dismutase mutations preferentially reduce the repulsive charge of the proteins. J. Biol. Chem. 282, 21230-21236 (2007).

46. Ayers, J. et al. Distinctive features of the D101N and D101G variants of superoxide dismutase 1; two mutations that produce rapidly progressing motor neuron disease. J. Neurochem. 128, 305-314 (2014).

47. Naini, A., Mehrazin, M., Lu, J., Gordon, P. \& Mitsumoto, H. Identification of a 
bioRxiv preprint doi: https://doi.org/10.1101/2021.12.07.471532; this version posted December 9, 2021. The copyright holder for this preprint

(which was not certified by peer review) is the author/funder, who has granted bioRxiv a license to display the preprint in perpetuity. It is made available under aCC-BY-NC-ND 4.0 International license.

novel D109Y mutation in $\mathrm{Cu} / \mathrm{Zn}$ superoxide dismutase $(\operatorname{sod} 1)$ gene associated with amyotrophic lateral sclerosis. J. Neurol. Sci. 254, 17-21 (2007). 

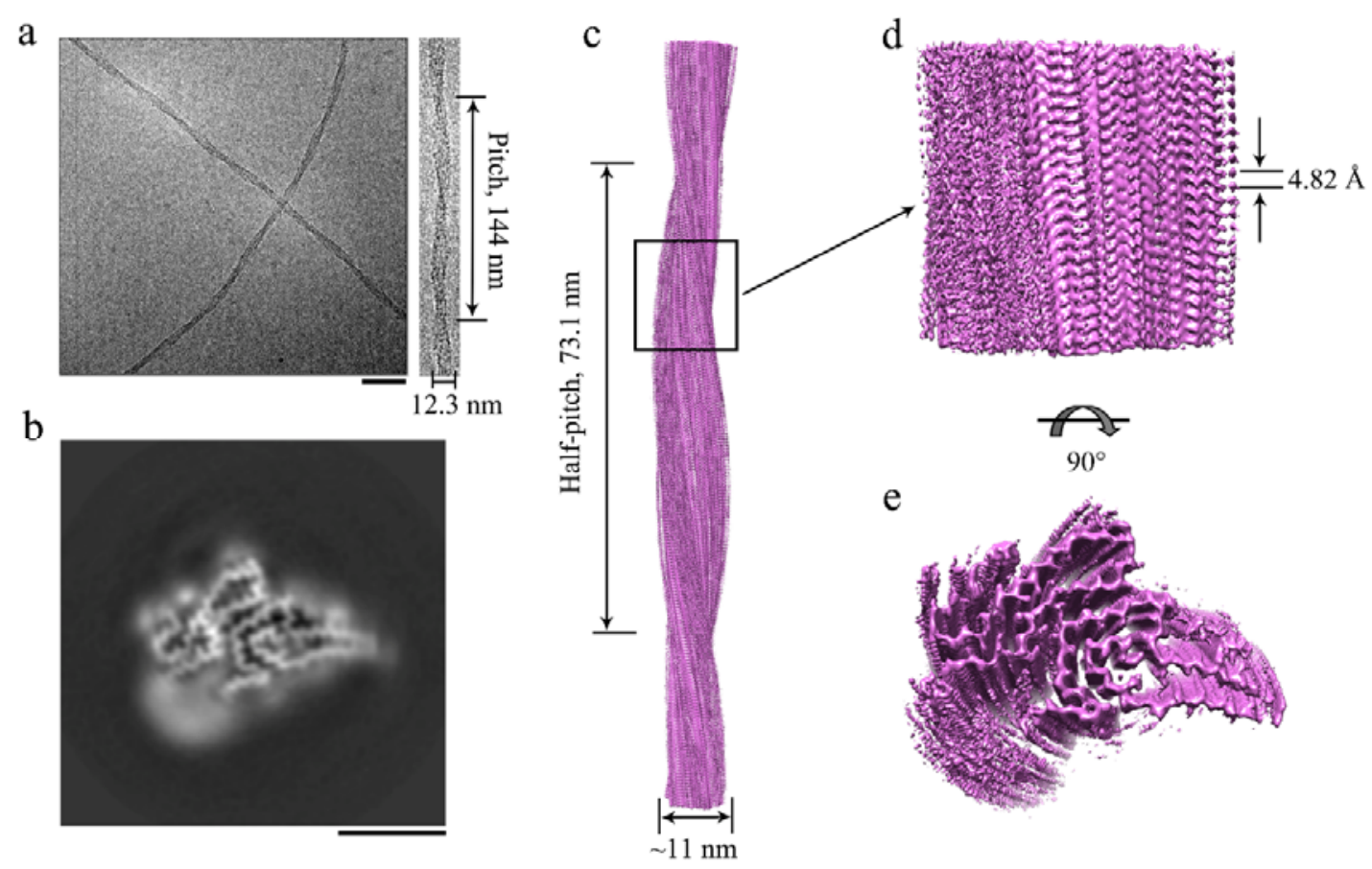

Fig. 1 | Cryo-EM structure of SOD1 fibrils. a, Raw cryo-EM image of amyloid fibrils assembled from recombinant, full-length apo human SOD1 under semi-reducing conditions. Enlarged section of (right) showing a single protofibril intertwined into a left-handed helix, with a fibril full width of $12.3 \pm 0.7 \mathrm{~nm}(n=8)$ and a helical pitch of $144 \pm 5 \mathrm{~nm}(n=8)$. The scale bar represents $50 \mathrm{~nm}$. The helical pitch and fibril width were measured and expressed as the mean \pm SD of values obtained in eight independent measurements. $\mathbf{b}$, Cross-sectional view of the 3D map of the SOD1 fibril showing a protofibril comprising the N-terminal part (left) and the C-terminal part (right), with an unstructured flexible region (bottom). Scale bars, 5 nm. c, 3D map showing a single protofibril (in orchid) intertwined into a left-handed helix, with a fibril core width of $\sim 11 \mathrm{~nm}$ and a half-helical pitch of $73.1 \mathrm{~nm}$. d, Enlarged section showing a side view of the density map. Close-up view of the density map in $\mathbf{c}$ showing that the subunit in a protofibril stacks along the fibril axis with a helical rise of $4.82 \AA$. e, Top view of the density map. 


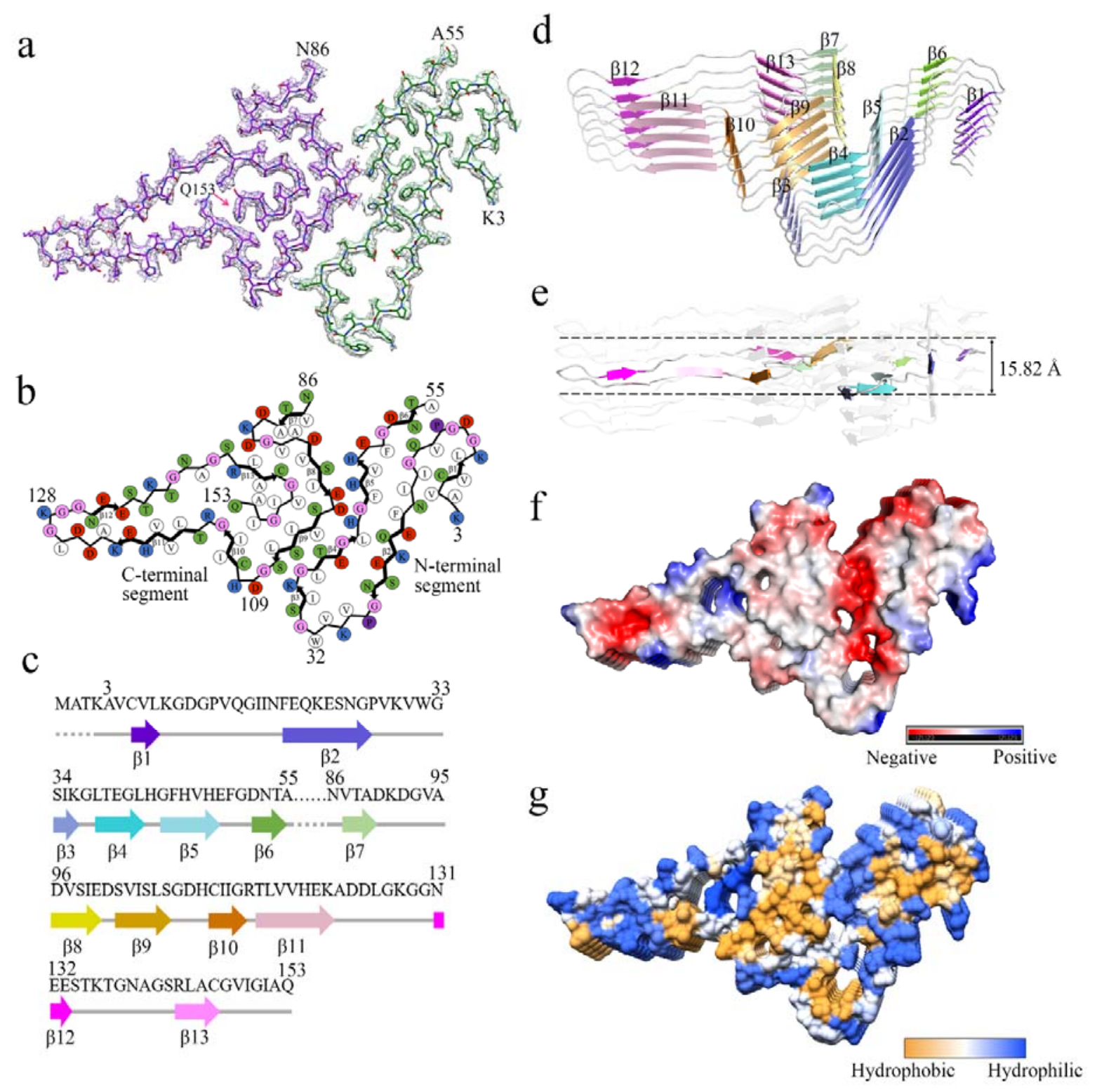

Fig. 2 | Atomic structure of SOD1 fibrils. a, Cryo-EM map of an amyloid fibril from recombinant, full-length apo human SOD1 with the atomic model overlaid. The SOD1 fibril core comprises the N-terminal segment (residues 3 to 55) and the C-terminal segment (residues 86 to 153 ) colored green and purple, respectively, with a structural break. b, Schematic view of the SOD1 fibril core. Residues are colored as follows: white, hydrophobic; green, polar; red and blue, negatively and positively charged, respectively; and magenta, glycine. $\beta$ strands are indicated with bold lines. Side chains of most hydrophobic residues are located in the interior of the SOD1 fibril fold. c, Sequence of the fibril core comprising residues 3 to 55 and 86 to 153 from 
full-length human SOD1 (1 to 153) with the observed six $\beta$ strands colored violet ( $\beta 1$ ), blue $(\beta 2)$, light blue $(\beta 3)$, cyan $(\beta 4)$, light cyan $(\beta 5)$, and green $(\beta 6)$ in the $\mathrm{N}$-terminal region and the observed seven $\beta$ strands colored light green $(\beta 7)$, yellow $(\beta 8)$, gold $(\beta 9)$, orange $(\beta 10)$, pink $(\beta 11)$, magenta $(\beta 12)$, and light magenta $(\beta 13)$ in the C-terminal region. The dotted lines correspond to residues 1 to 2 and residues 56 to 85 not modeled in the cryo-EM density. d, Ribbon representation of the structure of an SOD1 fibril core containing five molecular layers and two segments. e, As in $\mathbf{d}$, but viewed perpendicular to the helical axis, revealing that the height of one layer along the helical axis is $15.82 \AA$. f, Electrostatic surface representation of the structure of an SOD1 fibril core containing five molecular layers and two segments. g, Hydrophobic surface representation of the structure of an SOD1 fibril core as in $\mathbf{d}$. f,g, Three pairs of amino acids (Lys36 and Asp109; His43 and Asp101; and His46 and Glu100) form three salt bridges at the intramolecular interface between the $\mathrm{N}$ - and $\mathrm{C}$-terminal regions of SOD1 fibril. The surface of two regions of the SOD1 fibril core is shown according to the electrostatic properties (f) or the hydrophobicity (g) of the residues. 




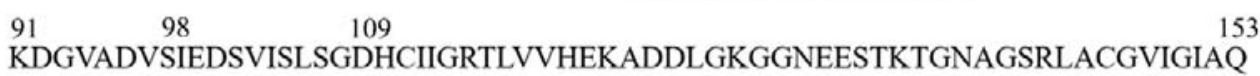

C-terminal half of the interface

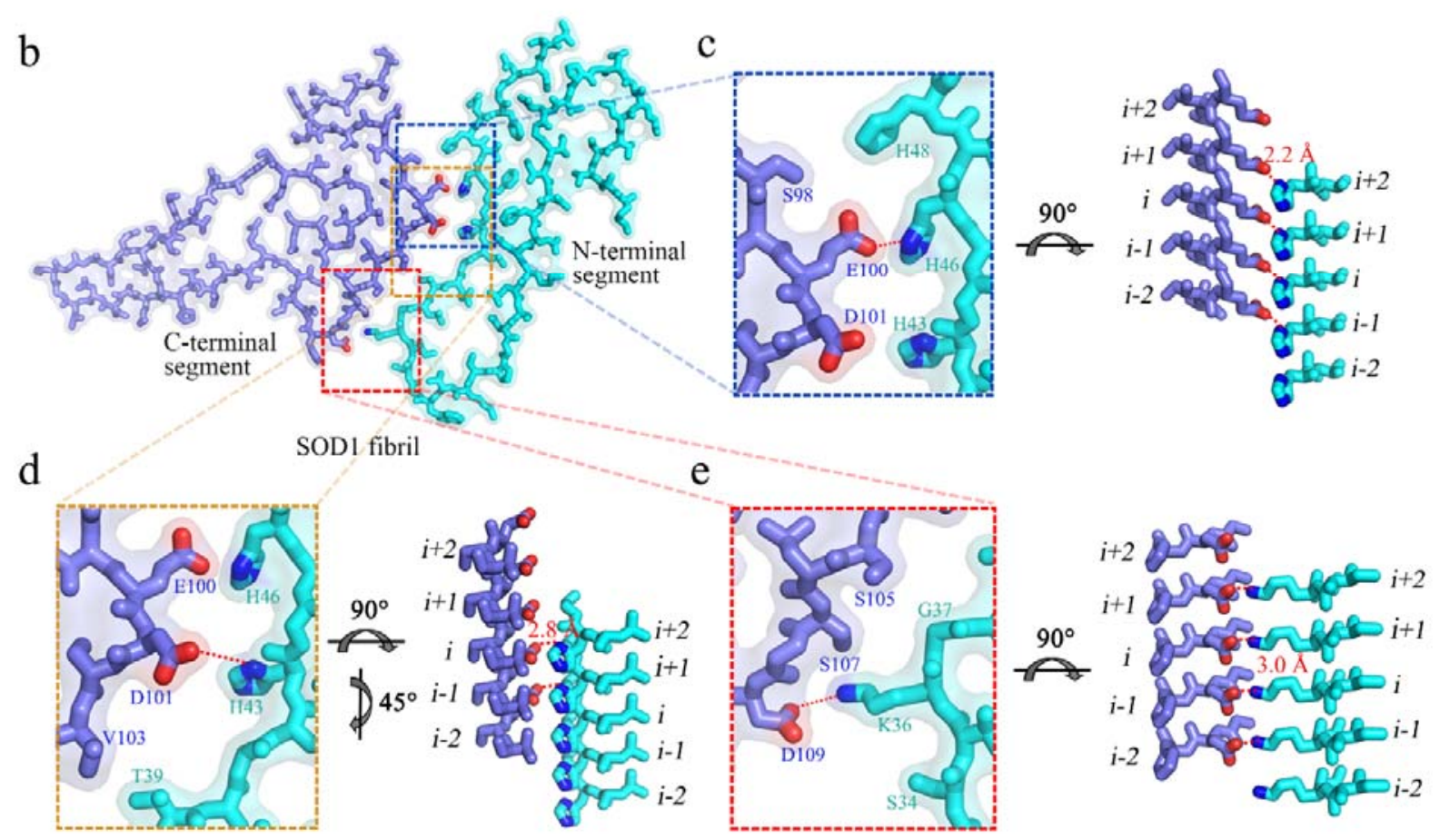

Fig. 3 | Close-up view of the intramolecular interface between the $N$ - and

C-terminal regions of SOD1 fibril. a, The primary sequence of the SOD1 fibril core.

The cyan bar marks the N-terminal half of the interface and the blue bar marks the C-terminal half. The dotted lines correspond to residues 56 to 85 not modeled in the cryo-EM density and represent a structural break between the $\mathrm{N}$ - and C-terminal regions of SOD1 fibril. b, A space-filled model overlaid onto stick representation of the SOD1 fibril, in which the N-terminal segment is shown in cyan and the C-terminal segment in blue. Lys/Asp pairs, His/Asp pairs, and His/Glu pairs that form salt bridges are highlighted in red (oxygen atoms in Asp and Glu) and blue (nitrogen atom in Lys and His), and the intramolecular interface is magnified in c to e. c-e, Magnified top views of the three regions of the intramolecular L-shaped interface between the $\mathrm{N}$ - and $\mathrm{C}$-terminal regions of SOD1 fibril, where three pairs of amino acids (Lys36 and Asp109; His43 and Asp101; and His46 and Glu100) form three salt 
bridges. Two side views (right) highlighting a strong salt bridge between Glu100 from the C-terminal segment $(i)$ and His46 from the adjacent N-terminal segment $(i+1)$, with a distance of $2.2 \AA$ (red), or between Asp101 from the molecular layer $(i-l)$ of the C-terminal region and His43 from the molecular layer $(i+2)$ of the N-terminal region, with a distance of $2.8 \AA$ (red). A side view (right) highlighting a strong salt bridge between Asp109 from the C-terminal part ( $i$ ) and Lys36 from the adjacent $\mathrm{N}$-terminal part $(i+1)$, with a distance of $3.0 \AA$ (red). 

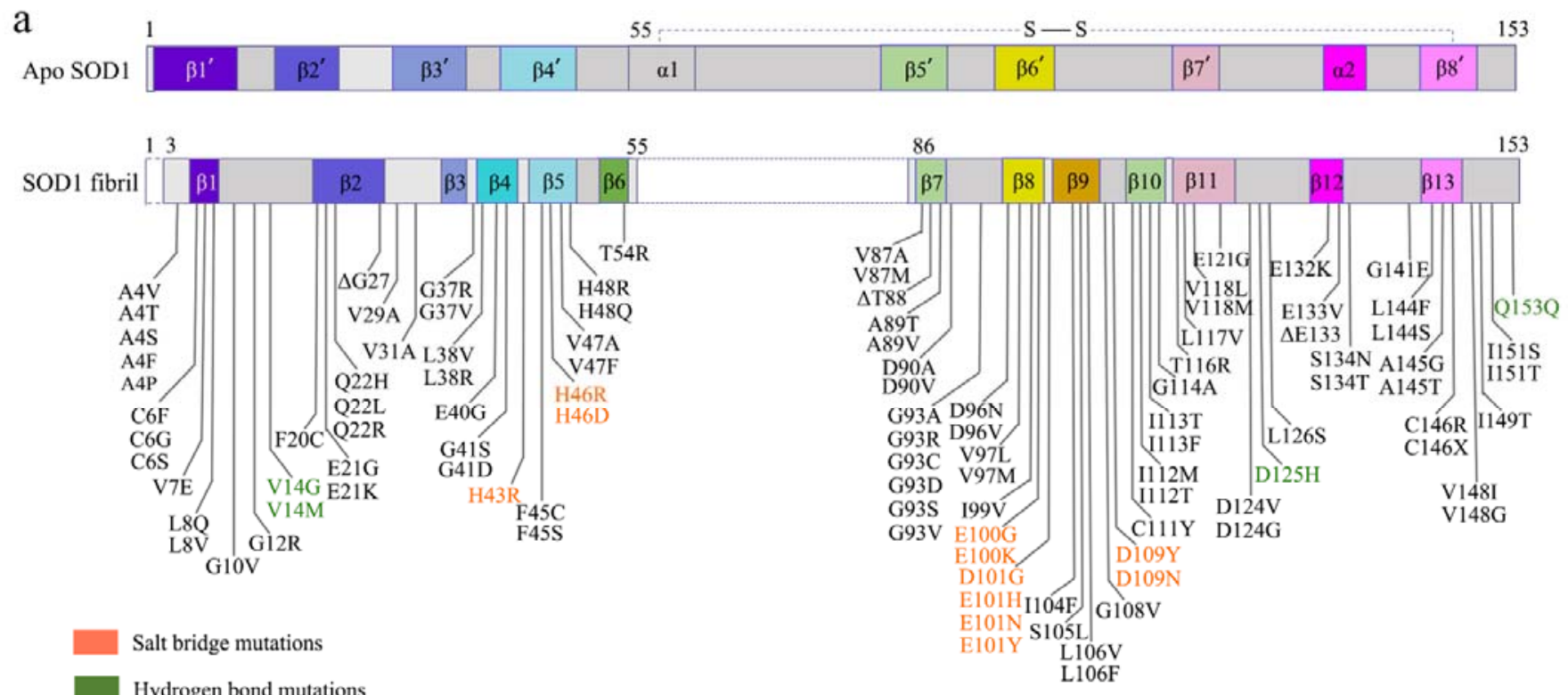

b

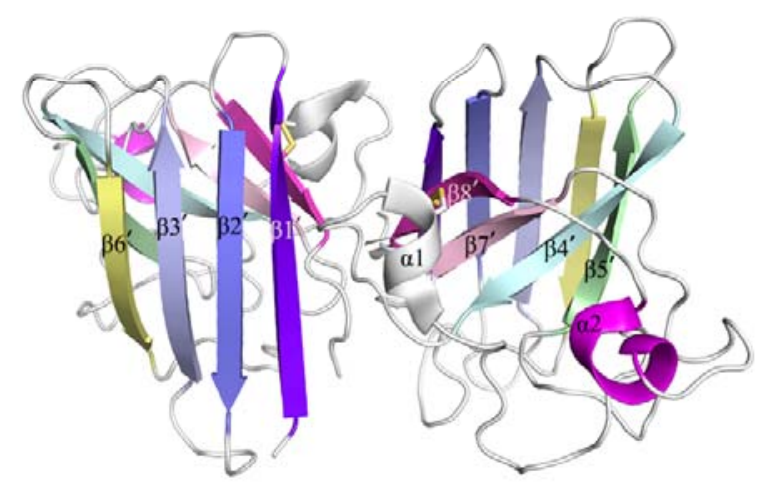

Apo SOD1 dimer

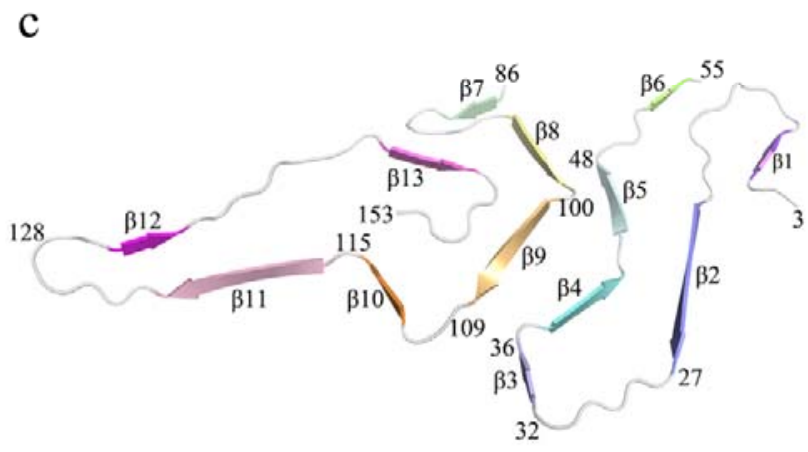

SOD1 fibril

Fig. 4 Comparison of the structures of apo SOD1 dimer and SOD1 fibril. a, Sequence alignment of the full-length apo human SOD1 (1 to 153) dimer with eight $\beta$-strands, two $\alpha$-helices, and a single disulfide bond between $\mathrm{Cys}^{57}$ in $\alpha 1$ and Cys ${ }^{146}$ in $\beta 8^{\prime}$ in each subunit (PDB $\left.1 \mathrm{HL} 4\right)^{12}$ and the SOD1 fibril core comprising residues 3 to 55 and 86 to 153 from full-length human SOD1 (1 to 153) with the observed thirteen $\beta$ strands. Dashed boxes correspond to residues 1 to 2 and residues 56 to 85 , which were not modeled in the cryo-EM density. Among two hundred and seventeen mutations linked to familial $\mathrm{ALS}^{1-5,8,20-25,28,29,32,42-47}$ (https://alsod.iop.kcl.ac.uk/), one hundred and eighty-two clinically identified mutations are located within the SOD1 fibril core structure, in which one hundred and five representative genetic ALS-related mutations are listed in (A). b, Ribbon representation of the structure of 
full-length apo human SOD1 (1 to 153) dimer with eight $\beta$-strands (to form a $\beta$-barrel), two $\alpha$-helices, and a disulfide bridge (yellow line) linking $\alpha 1$ and $\beta 8^{\prime}$ in each subunit (PDB 1HL4) ${ }^{12}$. c, Ribbon representation of the structure of a SOD1 fibril core containing one molecular layer and thirteen $\beta$-strands, in which the $\mathrm{N}$-terminal segment contains six $\beta$ strands ( $\beta 1$ to $\beta 6$ ) and the $C$-terminal segment contains seven $\beta$ strands ( $\beta 7$ to $\beta 13)$. 


\section{Table 1 | Cryo-EM data collection, refinement and validation statistics}

\begin{tabular}{|c|c|}
\hline & $\begin{array}{l}\text { SOD1 fibril } \\
\text { (EMD-32227, PDB } \\
\text { 7VZF) }\end{array}$ \\
\hline \multicolumn{2}{|l|}{ Data collection and processing } \\
\hline Magnification & 130,000 \\
\hline Voltage (kV) & 300 \\
\hline Camera & $\begin{array}{l}\text { K2 summit } \\
\text { (Titan Krios) }\end{array}$ \\
\hline Frame exposure time (s) & 0.16 \\
\hline Movie frames $(n)$ & 40 \\
\hline Electron exposure $\left(\mathrm{e}^{-} / \AA^{2}\right)$ & 60 \\
\hline Defocus range $(\mu \mathrm{m})$ & -2.0 to -1.2 \\
\hline Pixel size $(\AA)$ & 1.04 \\
\hline Symmetry imposed & $C 1$ \\
\hline Box size (pixel) & 320 \\
\hline Inter-box distance $(\AA)$ & 33.3 \\
\hline Micrographs collected $(n)$ & 2,931 \\
\hline Segments extracted $(n)$ & 288,744 \\
\hline Segments after Class2D $(n)$ & 147,525 \\
\hline Segments after Class3D $(n)$ & 70,067 \\
\hline Map resolution $(\AA)$ & 3.08 \\
\hline FSC threshold & 0.143 \\
\hline Map resolution range $(\AA)$ & $2.30-5.01$ \\
\hline \multicolumn{2}{|l|}{ Refinement } \\
\hline Initial model used & De novo \\
\hline Model resolution $(\AA ̊)$ & 3.08 \\
\hline FSC threshold & 0.143 \\
\hline Model resolution range $(\AA)$ & 3.08 \\
\hline Map sharpening $B$ factor $\left(\AA^{2}\right)$ & -97.89 \\
\hline \multicolumn{2}{|l|}{ Model composition } \\
\hline Nonhydrogen atoms & 2,628 \\
\hline Protein residues & 363 \\
\hline Ligands & 0 \\
\hline \multicolumn{2}{|l|}{$B$ factors $\left(\AA^{2}\right)$} \\
\hline Protein & 70.90 \\
\hline \multicolumn{2}{|l|}{ R.m.s. deviations } \\
\hline Bond lengths $(\AA)$ & 0.009 \\
\hline Bond angles $\left({ }^{\circ}\right)$ & 1.060 \\
\hline \multicolumn{2}{|l|}{ Validation } \\
\hline MolProbity score & 2.86 \\
\hline Clashscore & 37.29 \\
\hline Poor rotamers (\%) & 0 \\
\hline \multicolumn{2}{|l|}{ Ramachandran plot } \\
\hline Favored $(\%)$ & 73.50 \\
\hline Allowed (\%) & 26.50 \\
\hline Disallowed (\%) & 0 \\
\hline
\end{tabular}




\section{METHODS}

Protein purification. A plasmid-encoding, full-length human SOD1 (1-153) was a gift from Dr. Thomas O’Halloran (Chemistry of Life Processed Institute, Northwestern University). The sequence for SOD1 1-153 was expressed from the vector pET-3d, which was transformed into E. coli BL21 (DE3) cells (Novagen, Merck, Darmstadt, Germany). SOD1 protein was purified to homogeneity by Q-Sepharose chromatography as describe by Chattopadhyay et al. ${ }^{26}$ and Xu et al. ${ }^{6}$. After purification, recombinant wild-type SOD1 was demetallated by dialysis in 10 $\mathrm{mM}$ EDTA and $10 \mathrm{mM}$ NaAc buffer ( $\mathrm{pH}$ 3.8) five times as described by Chattopadhyay et al. ${ }^{26}$ and Xu et al. ${ }^{6} .10 \mathrm{mM}$ NaAc buffer (pH 3.8) and $20 \mathrm{mM}$ tris-HCl buffer $(\mathrm{pH} 7.4)$ were used for further dialysis. The apo SOD1 was then concentrated, filtered, and stored at $-80^{\circ} \mathrm{C}$. AAnalyst -800 atomic absorption spectrometer (PerkinElmer) was used to quantify metal content of SOD1 samples. Samples of wild-type SOD1 contained less than 5\% of residual metal ions, indicating that the samples were indeed in the apo state. SDS-PAGE and mass spectrometry were used to confirm that the purified apo SOD1 proteins were single species with an intact disulfide bond. A NanoDrop OneC Microvolume UV-Vis Spectrophotometer (Thermo Fisher Scientific) was used to determine the concentration of apo SOD1 according to its absorbance at $214 \mathrm{~nm}$ with a standard calibration curve drawn by BSA. 
SOD1 fibril formation. Recombinant, full-length apo human SOD1 $(30 \mu \mathrm{M})$ were incubated in $20 \mathrm{mM}$ tris-HCl buffer (pH 7.4) containing $5 \mathrm{mM}$ TCEP and shaking at $37{ }^{\circ} \mathrm{C}$ for 40 to 48 hours, and the SOD1 fibrils were collected. Large aggregates in SOD1 fibril samples were removed by centrifugation for $5000 \mathrm{~g}$ at $4{ }^{\circ} \mathrm{C}$ for $10 \mathrm{~min}$. The supernatants were then concentrated to $\sim 30 \mu \mathrm{M}$ in a centrifugal filter (Millipore). A NanoDrop OneC Microvolume UV-Vis Spectrophotometer (Thermo Fisher Scientific) was used to determine the concentrations of the SOD1 fibril according to its absorbance at $214 \mathrm{~nm}$ with a standard calibration curve drawn by BSA.

Congo red binding assays. SOD1 fibrils were analyzed by Congo red binding assays. A stock solution of $200 \mu \mathrm{M}$ Congo red was prepared in phosphate-buffered saline and filtered through a filter of $0.22-\mu \mathrm{m}$ pore size before use. In a typical assay, the SOD1 fibril sample was mixed with a solution of Congo red to yield a final Congo red concentration of $50 \mu \mathrm{M}$ and a final SOD1 concentration of $10 \mu \mathrm{M}$, and the absorbance spectrum between 400 and $700 \mathrm{~nm}$ was then recorded on a Cytation 3 Cell Imaging Multi-Mode Reader (BioTek).

TEM of SOD1 fibrils. SOD1 fibrils were examined by TEM of negatively stained samples. Ten microliters of SOD1 fibril samples $(\sim 30 \mu \mathrm{M})$ were loaded on copper grids for $30 \mathrm{~s}$ and washed with $\mathrm{H}_{2} \mathrm{O}$ for $10 \mathrm{~s}$. Samples on grids were then stained with $2 \%(\mathrm{w} / \mathrm{v})$ uranyl acetate for $30 \mathrm{~s}$ and dried in air at $25^{\circ} \mathrm{C}$. The stained samples were 
examined using a JEM-1400 Plus transmission electron microscope (JEOL) operating at $100 \mathrm{kV}$.

Cryo-EM of SOD1 fibrils. SOD1 fibrils were produced as described above. An aliquot of $3.5 \mu \mathrm{l}$ of $\sim 30 \mu \mathrm{M}$ SOD1 fibril solution was applied to glow-discharged holey carbon grids (Quantifoil $\mathrm{Cu}$ R1.2/1.3, 300 mesh), blotted for $3.5 \mathrm{~s}$ and plunge-frozen in liquid ethane using an FEI Vitrobot Mark IV. The grids were examined using an FEI Talos F200C microscope, operated at $200 \mathrm{kV}$, and equipped with a field emission gun and an FEI Ceta camera (Thermo Fisher Scientific). The cryo-EM micrographs were acquired on an FEI Titan Krios microscope operated at $300 \mathrm{kV}$ (Thermo Fisher Scientific) and equipped with a Gatan Bio-Quantum K2 Summit camera. A total of 2931 movies were collected by beam-image shift data collection methods ${ }^{48}$ in super-resolution mode at a nominal magnification of $\times 130,000$ (pixel size, $1.04 \AA$ ) and a dose of $9.375 \mathrm{e}^{-} \AA^{-2} \mathrm{~s}^{-1}$ (see Table 1). An exposure time of $6.4 \mathrm{~s}$ was used, and the resulting videos were dose-fractionated into 40 frames. A defocus range of -1.2 to $-2.0 \mu \mathrm{m}$ was used.

Helical reconstruction. All 40 video frames were aligned, summed, and dose-weighted by MotionCor2 and further binned to a pixel size of $1.04 \AA$ (ref. ${ }^{49}$ ). Contrast transfer function estimation of aligned, dose-weighted micrographs was performed by CTFFIND4.1.8 (ref. ${ }^{50}$ ). Subsequent image-processing steps, which 
include manual picking, particle extraction, 2D and 3D classifications, 3D refinement, and post-processing, were performed by RELION3.1 (ref. ${ }^{39}$ ).

In total, 10,488 fibrils were picked manually from 2931 micrographs, and 686- and 320-pixel boxes were used to extract particles by $90 \%$ overlap scheme. Two-dimensional classification of 686-box size particles was used to calculate the initial twist angle. In regard to helical rise, $4.8 \AA$ was used as the initial value. Particles were extracted into 320-box sizes for further processing. After several iterations of $2 \mathrm{D}$ and $3 \mathrm{D}$ classifications, particles with the same morphology were picked out. Local searches of symmetry in 3D classification were used to determine the final twist angle and rise value. The $3 \mathrm{D}$ initial model was built by selected $2 \mathrm{D}$ classes; 3D classification was performed several times to generate a proper reference map for 3D refinement. Three-dimensional refinement of the selected 3D classes with appropriate reference was performed to obtain final reconstruction. The final map of SOD1 fibrils was convergent with a rise of $4.82 \AA$ and a twist angle of $-1.187^{\circ}$. Postprocessing was preformed to sharpen the map with a $B$ factor of $-97.89 \AA^{2}$. On the basis of the gold standard Fourier shell correlation $(\mathrm{FSC})=0.143$ criteria, the overall resolution was reported as $3.08 \AA$. The statistics of cryo-EM data collection and refinement is shown in Table 1.

Atomic model building and refinement. COOT $^{51}$ was used to build de novo and modify the atomic model of the SOD1 fibril. The model with three adjacent layers 
was generated for structure refinement. The model was refined using the real-space refinement program in PHENIX $^{52}$.

\section{Reporting summary}

Further information on experimental design is available in the Nature Research Reporting Summary linked to this article.

\section{Data availability}

Cryo-EM density maps and the atomic model of human SOD1 fibrils are available through the Electron Microscopy Data Bank and Protein Data Bank with accession codes EMD-32227 and PDB 7VZF, respectively. The source data underlying Extended Data Fig. 1b are provided as a Source Data.

48. Wu, C., Huang, X., Cheng, J., Zhu, D. \& Zhu, X. High-quality, high-throughput cryo-electron microscopy data collection via beam tilt and astigmatism-free beam-image shift. J. Struct. Biol. 208, 107396 (2019).

49. Zheng, S. Q. et al. MotionCor2: anisotropic correction of beam-induced motion for improved cryo-electron microscopy. Nat. Methods 14, 331-332 (2017).

50. Rohou, A. \& Grigorieff, N. CTFFIND4: Fast and accurate defocus estimation from electron micrographs. J. Struct. Biol. 192, 216-221 (2015). 
51. Emsley, P., Lohkamp, B., Scott, W. G. \& Cowtan, K. Features and development of Coot. Acta Crystallogr. D Biol. Crystallogr. 66, 486-501 (2010).

52. Adams, P. D. et al. PHENIX: a comprehensive Python-based system for macromolecular structure solution. Acta Crystallogr. D Biol. Crystallogr. 66, 213-221 (2010).

\section{Acknowledgements}

Y.L. and C.L. acknowledge funding from the National Natural Science Foundation of China (no. 31770833) and the Major State Basic Research Development Program (no. 2019YFE0120600). Y.L. also acknowledges financial support from the National Natural Science Foundation of China (nos. 32071212 and 31570779), the Key Project of Basic Research, Science and Technology R\&D Fund of Shenzhen (no. JCYJ20200109144418639), and the Translational Medicine and Interdisciplinary Research Joint Fund of Zhongnan Hospital of Wuhan University (no. ZNJC201934). C.L. was also supported by the National Natural Science Foundation of China (nos. 91853113 and 31872716), the Science and Technology Commission of Shanghai Municipality (nos. 18JC1420500, 20XD1425000, and 2019SHZDZX02) and the CAS project for Young Scientists in Basic research (no. YSBR-009). L.-Q.W. acknowledges financial support from China Postdoctoral Science Foundation (nos. 2021TQ0252 and 2021M700103). P.Y. acknowledges financial support from the Major State Basic Research Development Program (no. 2018YFA0507700) and the National Natural Science Foundation of China (no. 31722017). L.Z. acknowledges 
financial support from the Key Project of Basic Research, Science and Technology R\&D Fund of Shenzhen (no. JCYJ20200109144418639). Cryo-EM data were collected at the Center for Biological Imaging, Institute of Biophysics, Chinese Academy of Sciences, China. We thank T. V. O'Halloran (Northwestern University) for the gift of the human SOD1 plasmid; B. Zhu (Center for Biological Imaging, Institute of Biophysics, Chinese Academy of Sciences), X. Li (Center for Biological Imaging, Institute of Biophysics, Chinese Academy of Sciences), L. Wu (Center of Cryo Electron Microscopy, Zhejiang University), and F. Sun (Institute of Biophysics, Chinese Academy of Sciences) for technical assistance with cryo-EM; and Y. Wang (Institute of Biophysics, Chinese Academy of Sciences) for helpful suggestions.

Author contributions L.Z., P.Y., C.L. and Y.L. supervised the project. L.-Q.W., C.L. and Y.L. designed the experiments. L.-Q.W., H.-Y.Y., M.-Y. Z., X.H., W.-C.X. and J.C. purified human SOD1 and the SOD1 fibrils. L.-Q.W., H.-Y.Y. and M.-Y. Z. performed Congo red binding assays of SOD1 fibrils. L.-Q.W., Y.M., H.-Y.Y., K.Z., Q.W., D.Z. and D.L. collected, processed, and/or analyzed cryo-EM data. L.-Q.W., Y.M., C.L. and Y.L. wrote the manuscript. All authors proofread and approved the manuscript.

Competing interests The authors declare no competing interests. 

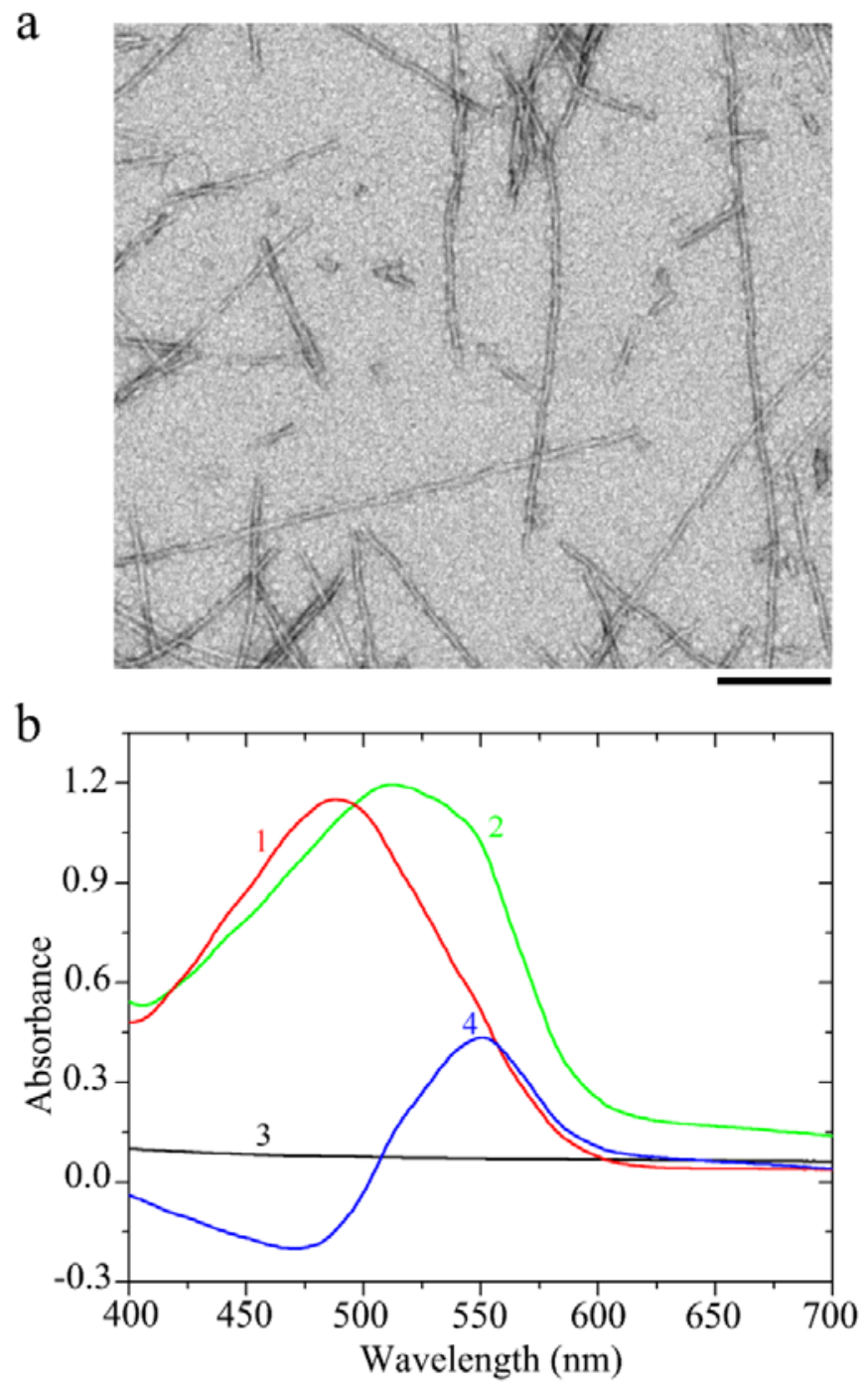

Extended Data Fig. 1 | SOD1 forms typical amyloid fibrils under semi-reducing conditions. a, A negative-staining TEM image of amyloid fibrils assembled from recombinant, full-length apo human SOD1 in $20 \mathrm{mM}$ tris- $\mathrm{HCl}$ buffer (pH 7.4) containing $5 \mathrm{mM}$ TCEP. Scale bar, $200 \mathrm{~nm}$. b, Amyloid fibrils from recombinant, full-length apo human SOD1 analyzed by Congo red binding assays. The difference spectra (Curve 4, blue) with the maximum absorbance at $550 \mathrm{~nm}$ were obtained by subtracting the absorbance spectra of SOD1 fibrils alone (Curve 3, black) and Congo red alone (Curve 1, red) with the maximum absorbance at $490 \mathrm{~nm}$ from those of SOD1 fibrils + Congo red (Curve 2, green). Congo red binding assays were carried out at $37^{\circ} \mathrm{C}$. These experiments were repeated three times with different batches of fibrils and similar results. Data behind graphs are available as source data. 


\section{a}

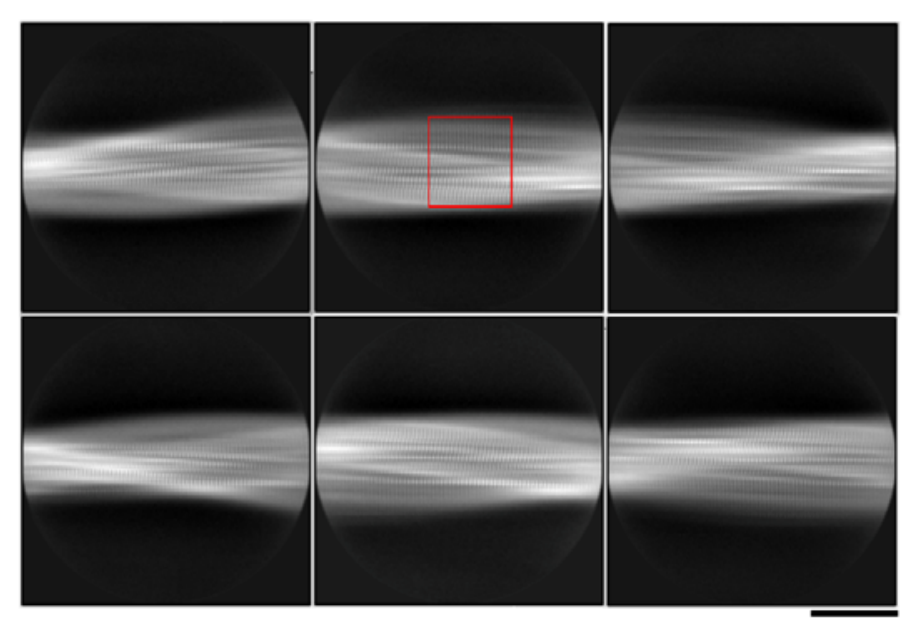

b

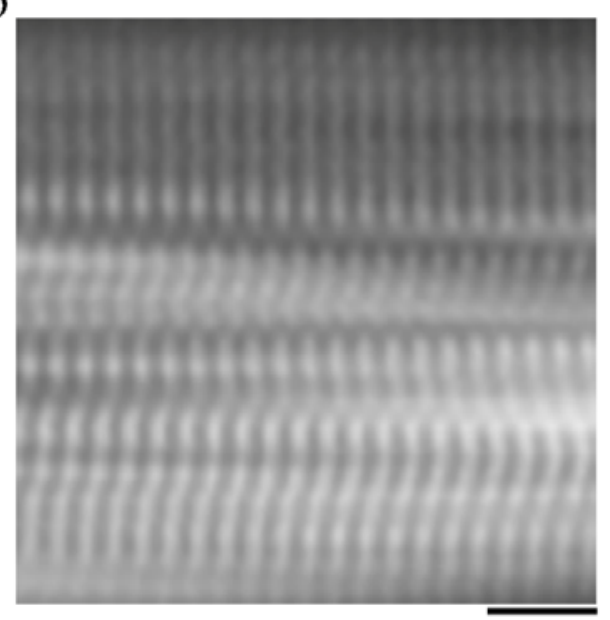

Extended Data Fig. 2 | Cryo-EM images of PrPfibrils. a, Cryo-EM micrographs of amyloid fibrils of full-length human PrP showing two protofibrils intertwined into a left-handed helix. Scale bar, $50 \mathrm{~nm}$. b, Reference-free 2D class averages of PrP fibrils showing two protofibrils intertwined. Scale bar, $10 \mathrm{~nm}$. c, Enlarged image of (b) showing two protofibrils arranged in a staggered manner. Scale bar, $2 \mathrm{~nm}$. 


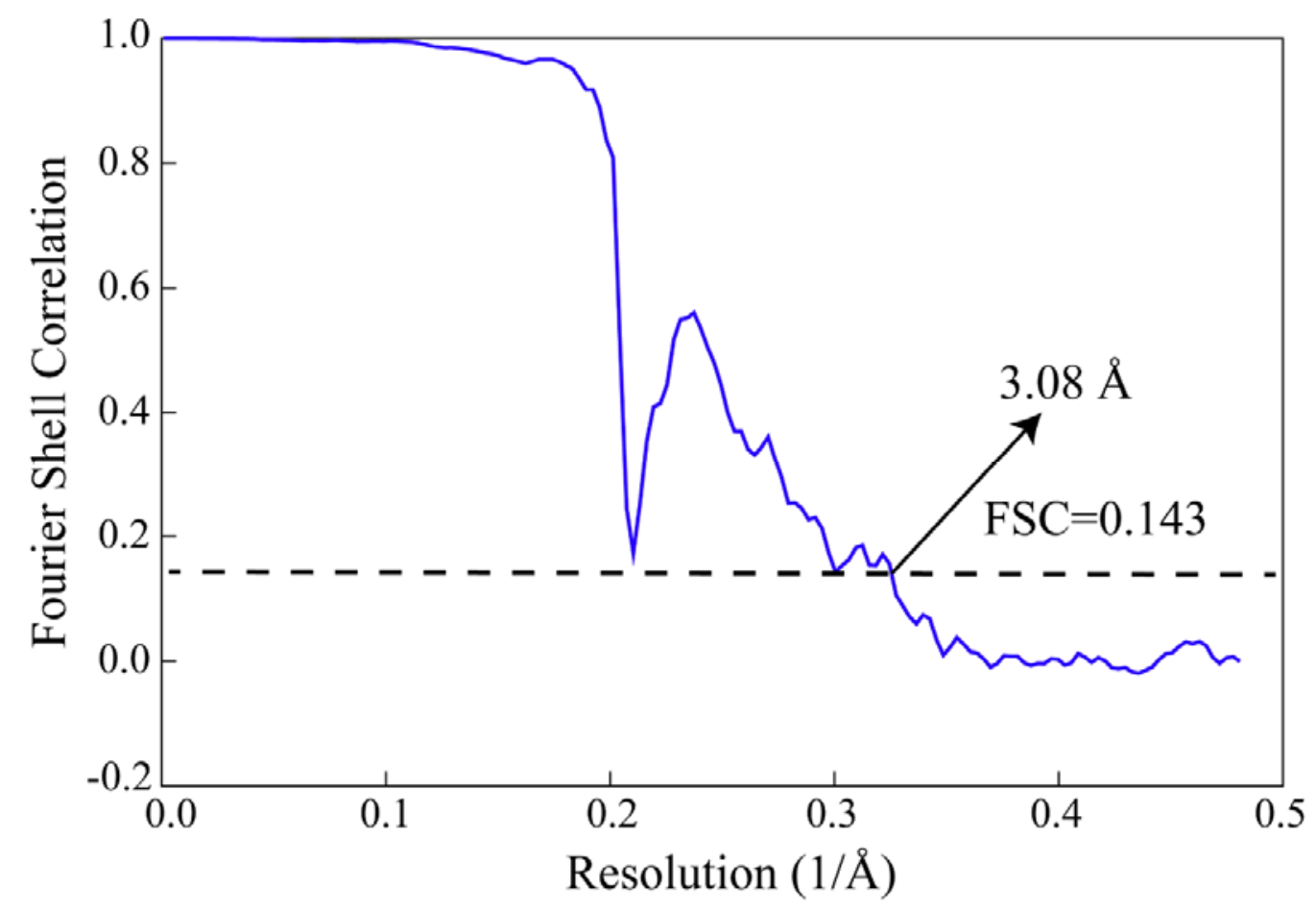

Extended Data Fig. 3 | Global resolution estimate for the SOD1 fibril reconstructions. Gold-standard refinement was used for estimation of the density map resolution. The global resolution of $3.08 \AA$ was calculated using a Fourier shell correlation (FSC) curve cut-off at 0.143 . 

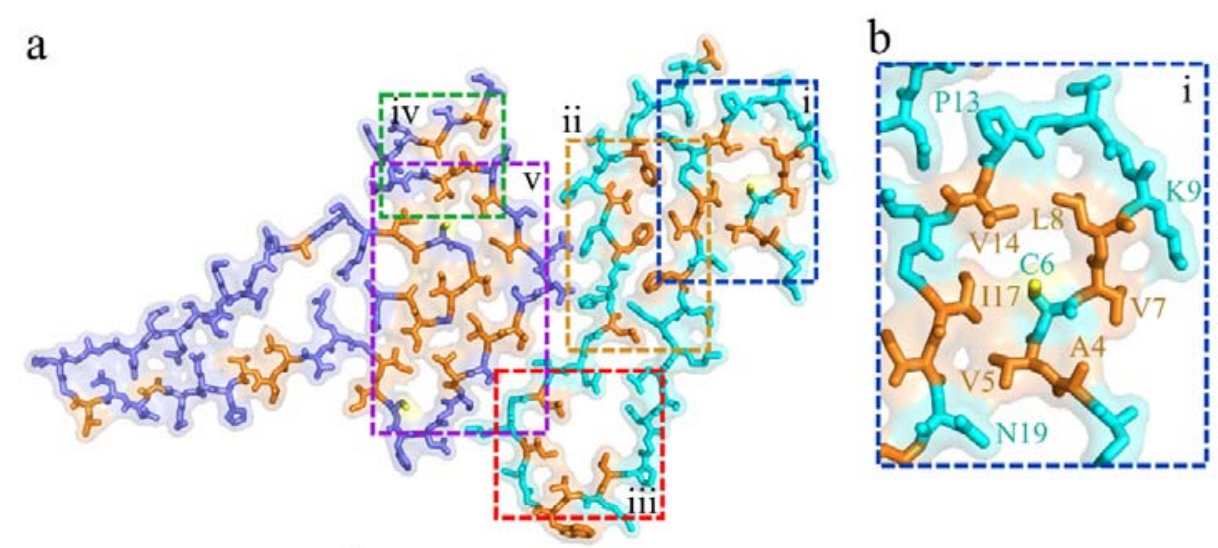

$\mathrm{c}$

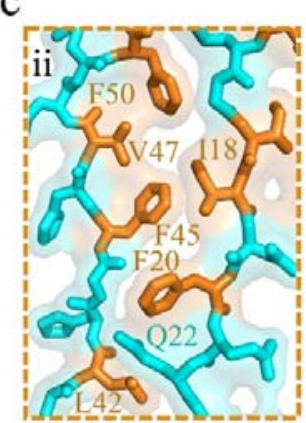

d

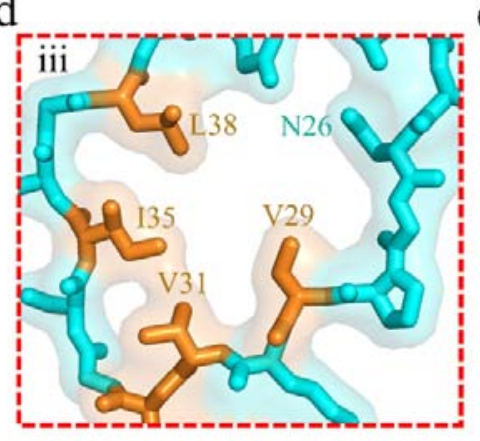

e

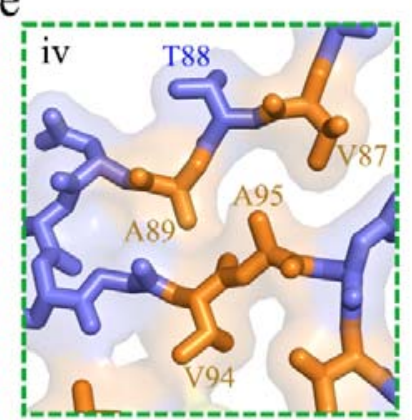



Extended Data Fig. 4 | Close-up view of the stick representation of the structure

of the SOD1 fibril stabilized by five hydrophobic cavities. a, A space-filled model

overlaid onto stick representation of the SOD1 fibril, in which the N-terminal part is shown in cyan and the C-terminal part in blue. Hydrophobic residues and sulfur atoms in Cys146 and Cys111 are highlighted in orange and yellow, respectively, and five hydrophobic cavities ( $\mathrm{i}$ to $\mathrm{v}$ ) in the SOD1 fibril are magnified in $\mathbf{b}$ to $\mathbf{f}$. b-f, Magnified top views of the five hydrophobic cavities in the SOD1 fibril, where hydrophobic side chains of Val5, Leu8, Val14, and Ile17, hydrophobic side chains of Ile18, Phe20, Leu42, Phe45, Val47, and Phe50, and hydrophobic side chains of Val29, Leu31, Ile35, and Leu38 are located in the interior of the N-terminal segment of SOD1 fibril to form stable hydrophobic cores, and hydrophobic side chains of Val87, Ala89, and Ala95 and hydrophobic side chains of Va194, Va197, Ile99, Ile104, Leu106, Ile113, Leu144, Ala145, Val148, Ile149, Ile151, and Ala152 are located in the interior of the C-terminal segment to form stable hydrophobic cores. 


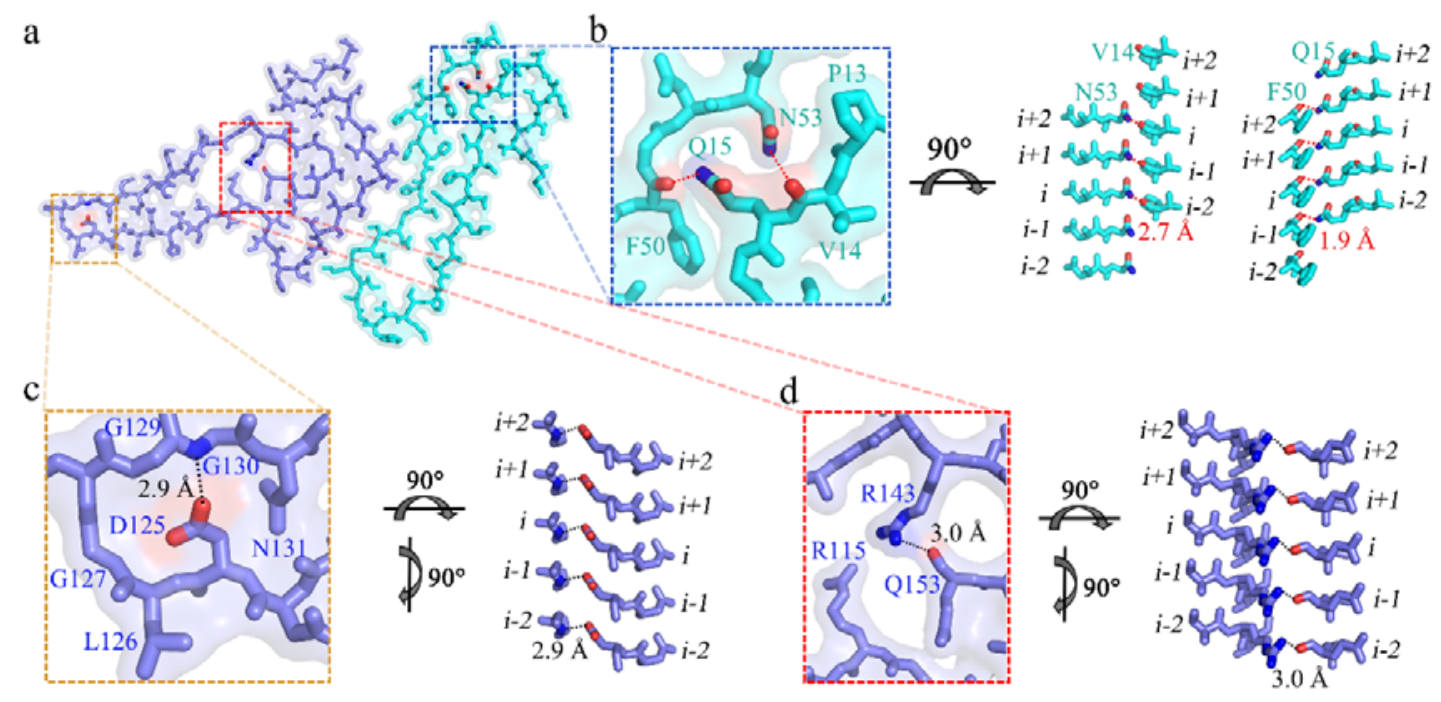

Extended Data Fig. 5 | Close-up view of the stick representation of the structure of the SOD1 fibril stabilized by four hydrogen bonds. a, A space-filled model overlaid onto stick representation of the SOD1 fibril, in which the N-terminal segment is shown in cyan and the C-terminal segment in blue. Val/Asn pairs, Phe/Gln pairs, Asp/Gly pairs, and Gln/Arg pairs that form hydrogen bonds are highlighted in red (oxygen atoms in Val, Phe, Asp, and Gln) and blue (nitrogen atoms in Asn, Gln, Gly, and Arg), and three hydrogen bond regions are magnified in $\mathbf{b}$ to $\mathbf{d}$. $\mathbf{b}-\mathbf{d}$, Magnified top views of the three hydrogen bond regions of the SOD1 fibril, where two pairs of amino acids (Val14 and Asn53; and Gln15 and Phe50) from the N-terminal part form two hydrogen bonds, and two pairs of amino acids (Gly130 and Asp125; and Arg143 and Gln153) from the C-terminal part also form two hydrogen bonds. Two side views (right) highlighting a hydrogen bond between Asn53 from the N-terminal part (i) and the main chain of Val14 from the molecular layer $(i-2)$ of the $\mathrm{N}$-terminal part, with a distance of $2.7 \AA$ (red), or between Gln15 from the N-terminal segment $(i)$ and the main chain of Phe50 from the adjacent $\mathrm{N}$-terminal segment $(i-1)$, with a distance of $1.9 \AA$ (red). Two side views (right) highlighting a hydrogen bond between Asp125 from the C-terminal part $(i)$ and the main chain of Gly130 from the same layer of the C-terminal part $(i)$, with a distance of $2.9 \AA$ (black), or between Arg143 from the C-terminal segment $(i)$ and the main chain of Gln153 from the same layer of the C-terminal segment $(i)$, with a distance of $3.0 \AA$ (black). 
a



$\mathrm{C}$

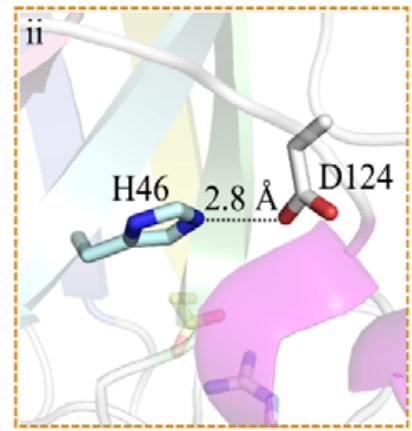

b

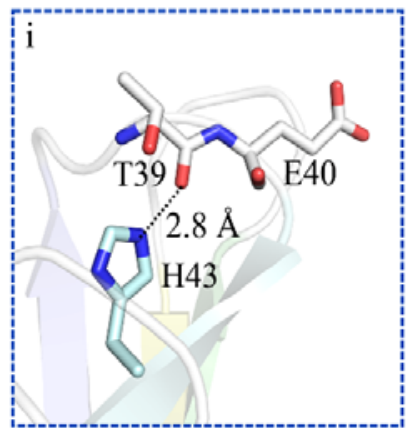

d

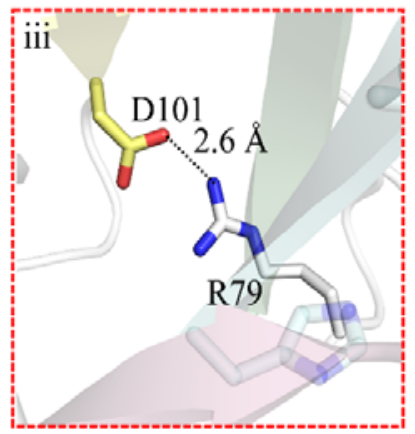

Extended Data Fig. 6 | Close-up view of the stick representation of the structure of apo SOD1 dimer. a, Ribbon representation of the structure of full-length apo human SOD1 (1 to 153) dimer with eight $\beta$-strands (to form a $\beta$-barrel) and two $\alpha$-helices in each subunit (PDB 1HL4) ${ }^{12}$, and Thr39, Glu40, His43, His46, Arg79, Asp101, and Asp124 in the right monomer are highlighted in stick representation. Thr/His pairs, His/Asp pairs, and Arg/Asp pairs that form hydrogen bonds and salt bridges are highlighted in red (oxygen atoms in Thr, Glu, and Asp) and blue (nitrogen atoms in His and Arg), and three hydrogen bond/salt bridge regions (i to iii) are magnified in $\mathbf{b}$ to $\mathbf{d}$. $\mathbf{b}-\mathbf{d}$, Magnified top views of the three regions of the apo human SOD1 monomer, where Thr39 and His43 form a hydrogen bond and two pairs of amino acids (His46 and Asp124; and Arg79 and Asp101) form two salt bridges. Two side views (right) highlighting a hydrogen bond between His43 and the main chain of Thr39, with a distance of $2.8 \AA$ A. Two side views (right) highlighting a strong salt bridge between Asp124 and His46, with a distance of $2.8 \AA$, or between Asp101 and Arg79, with a distance of $2.6 \AA$. 